\title{
Correcting for Perspective Distortion in Electroluminescence Images of Photovoltaic Panels
}

Mantel, Claire; Spataru, Sergiu; Parikh, Harsh; Sera, Dezso; Benatto, Gisele Alves dos Reis; Riedel, Nicholas; Thorsteinsson, Sune; Poulsen, Peter Behrensdorff; Forchhammer, Søren

\section{Published in:}

Proceedings of 7th World Conference on Photovoltaic Energy Conversion

Link to article, DOI:

10.1109/PVSC.2018.8547724

Publication date:

2018

Document Version

Peer reviewed version

Link back to DTU Orbit

Citation (APA):

Mantel, C., Spataru, S., Parikh, H., Sera, D., Benatto, G. A. D. R., Riedel, N., Thorsteinsson, S., Poulsen, P. B., \& Forchhammer, S. (2018). Correcting for Perspective Distortion in Electroluminescence Images of Photovoltaic Panels. In Proceedings of 7th World Conference on Photovoltaic Energy Conversion IEEE. https://doi.org/10.1109/PVSC.2018.8547724

\section{General rights}

Copyright and moral rights for the publications made accessible in the public portal are retained by the authors and/or other copyright owners and it is a condition of accessing publications that users recognise and abide by the legal requirements associated with these rights.

- Users may download and print one copy of any publication from the public portal for the purpose of private study or research.

- You may not further distribute the material or use it for any profit-making activity or commercial gain

- You may freely distribute the URL identifying the publication in the public portal 


\title{
Correcting for Perspective Distortion in Electroluminescence Images of Photovoltaic Panels
}

\author{
Claire Mantel $^{1}$, Sergiu Spataru ${ }^{2}$, Harsh Parikh ${ }^{2}$, Dezso Sera ${ }^{2}$, Gisele A. dos Reis Benatto ${ }^{1}$, Nicholas \\ Riedel $^{1}$, Sune Thorsteinsson ${ }^{1}$, Peter B. Poulsen ${ }^{1}$, Søren Forchhammer ${ }^{1}$ \\ ${ }^{1}$ DTU Fotonik, Kongens Lyngby, 2800, Denmark \\ ${ }^{2}$ Aalborg University, Aalborg, 9220, Denmark
}

\begin{abstract}
With the significant growth in the number of photovoltaic (PV) installations and their size, regular PV system inspection has become a challenge. Aerial drone imaging, based on visual, thermographic, and more recently luminescence, can be viable solutions for $P V$ inspection. However, to achieve effective detection and quantification of failure based on images acquired form Unmanned Aerial Vehicle, there is need for image quality enhancement and correction of distortions, inherent to the drone measurement process.

In this work we propose methods to automatically correct the perspective distortion in electroluminescent (EL) images of PV panels. We identified two main cases of perspective distortion: when the imaging plane is parallel to the panel plane or not, and propose methods to correct both. For both cases, the proposed method yields good results, as assessed by visual evaluation.
\end{abstract}

\section{INTRODUCTION}

With the global installed solar photovoltaic (PV) capacity estimated to reach $400 \mathrm{GW}$ in 2017 [1], there are already approximately 2 billion PV panels deployed worldwide. In this context, PV maintenance inspections pose a practical challenge, especially in large MW-level PV plants.

Recent developments and cost reductions in unmanned aerial vehicles (UAV) [2], enabled PV imaging inspection methods, such as visual and thermal infrared (IR), as a promising solution for fast and accurate PV plant inspection [3]. In addition, near-infrared/electroluminescence (EL) PV imaging techniques, one of the main laboratory PV panel inspection tools, have been successfully implemented for ground based PV system inspection [4]. Currently research is ongoing for implementing them in UAVs [5].

Although PV imaging tools can be used to inspect many panels in short time, they are still very much qualitative diagnostic techniques. Detecting, quantifying, reporting PV panel failures, from tens of thousands of panel images, requires automated image analysis to be feasible and cost efficient. One of the main sources of errors in automated image analysis are panel image distortions [6], inherent to the acquisition process. For example, in outdoor on-site imaging, be it ground-based or aerial inspection, the location and orientation of the camera can lead to an image in which the panel geometry is distorted due to perspective effect. This is illustrated in Fig. 1.
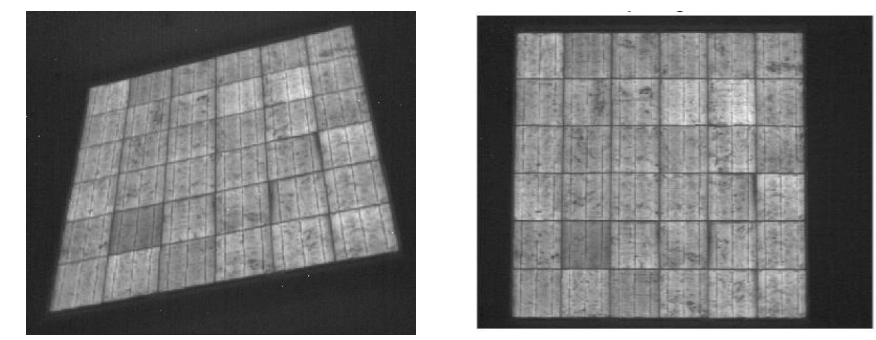

Fig. 1 Example of perspective distorted (left) and not (right) EL images of a Solvis SV36-150 PV panel, acquired with a SWIR InGaAs (640x512) camera.

In this work we propose methods to automatically correct the perspective distortion of PV panels in EL images, by estimating the panel rotation angles in the original image (Fig. 1 - left), relative to the normal perspective (Fig. 1-right), and apply a perspective transform.

First, we define the perspective distortion and desired normal perspective into mathematical terms. Then,

We identified two perspective distortion sub-cases, that required different correction approaches. In this work, we propose and validate methods for solving both cases.

Although we apply the perspective correction methodology to EL images, the methods presented have potential applicability for correcting the perspective of visual and IR images as well, which will be the focus of future work.

\section{PROBLEM DEFINITION}

The problem can be described in the following way:

Let $\mathrm{C}_{\mathrm{i}}=\left(\mathrm{x}_{\mathrm{Ci}}, \mathrm{y}_{\mathrm{Ci}}, \mathrm{z}_{\mathrm{Ci}}\right), \mathrm{i} \in\{1,2,3,4\}$ be the four corners of the considered panel in $\mathbb{R}^{3}$. As a panel is flat, these corners belong to one single plane $\wp_{1}$. Let $\wp_{2}$ be the plane of the image. The projections of the four corners of the panel are named $\mathrm{D}_{\mathrm{i}}=\left(\mathrm{x}_{\mathrm{Di}}, \mathrm{y}_{\mathrm{Di}}, \mathrm{z}_{\mathrm{Di}}\right), \mathrm{i} \in\{1,2,3,4\}$. The coordinates of the four corners $D_{i}, i \in\{1,2,3,4\}$ are "available information" and the aim of this paper is to retrieve the coordinates $C_{i}, i$ $\epsilon\{1,2,3,4\}$.

From the fundamental theorem of projective geometry [7], there exist a transformation $\mathrm{H}$ such as $\mathrm{C}_{\mathrm{i}}=\mathbf{H D}_{\mathrm{i}}$, with

$$
\mathbf{H}=\left[\begin{array}{lll}
h_{11} & h_{12} & h_{13} \\
h_{21} & h_{22} & h_{23} \\
h_{31} & h_{32} & h_{33}
\end{array}\right],
$$

since $\mathbf{H}$ is defined up to a scaling factor, let us assume $h_{33}=$ 1 . This can be seen as a parameter search problem, i.e. solving for the 8 degrees of freedom of $\mathbf{H}$. 
We distinguish two cases: when the two planes $\wp_{1}$ and $\wp_{2}$ are parallel or when they intersect. Those two cases correspond to two sub-problems and are each detailed below.

\section{A. Case 1 - Parallel planes}

In this case the two planes $\wp_{1}$ and $\wp_{2}$ are parallel, meaning that the panel was imaged orthogonally to the panel plane.

$\mathbf{H}$ belongs then to the class of isometries (roto translations), i.e. it can be expressed as:

$$
H=\left[\begin{array}{ccc}
\cos (\alpha) & -\sin (\alpha) & t_{\mathrm{x}} \\
-\sin (\alpha) & \cos (\alpha) & t_{\mathrm{y}} \\
0 & 0 & 1
\end{array}\right]
$$

In this paper, we disregard the translation aspect as the purpose is perspective distortion and not registration, i.e. only $\alpha$ is to be estimated. Therefore, the only issue for perspective distortion left in this scenario is a rotation along the Roll angle, i.e. along the axis orthogonal to the planes $\wp_{1}$ and $\wp_{2}$.

\section{B. Case 2 - Non-parallel planes}

In that case, there is none of the above assumptions on the $\mathbf{H}$ transformation between the $D_{i}, i \in\{1,2,3,4\}$ and the $C_{i}, i$ $\epsilon\{1,2,3,4\}$ points and the height variables $h_{i i}$ are to be determined.

\section{Proposed Method for CASE 1 - PARAllel Planes}

In this Section, it is assumed that the imaging plane $\wp_{2}$ is parallel to the panel plane $\wp_{1}$. As explained in Sec. II.A, in this case the only parameter to be estimated is the angle of the rotation $\alpha$. An example of such a case is illustrated in Fig. 2.

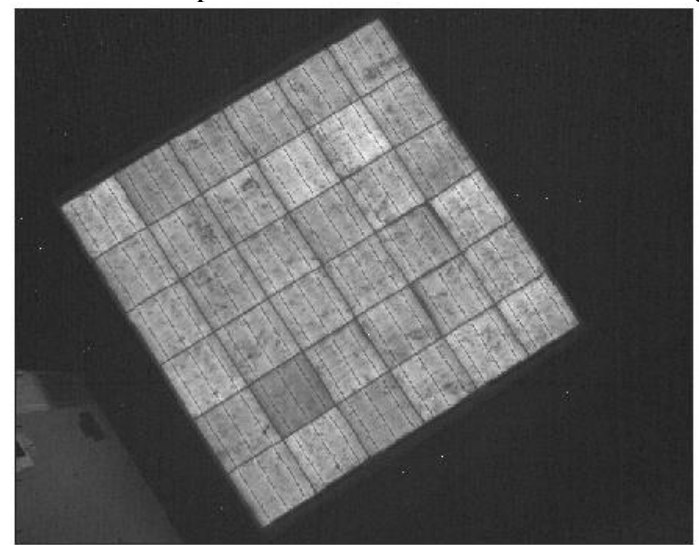

Fig. 2 Example of perspective distortion when imaging plane is parallel to PV panel plane

The proposed method consists in rotating the image by various angles and selecting the most probable based on the projection on the $\mathrm{X}$ and $\mathrm{Y}$ axes of the image (in is thus inspired by the Hough transform). The overall scheme of the proposed method is illustrated in Fig. 3.

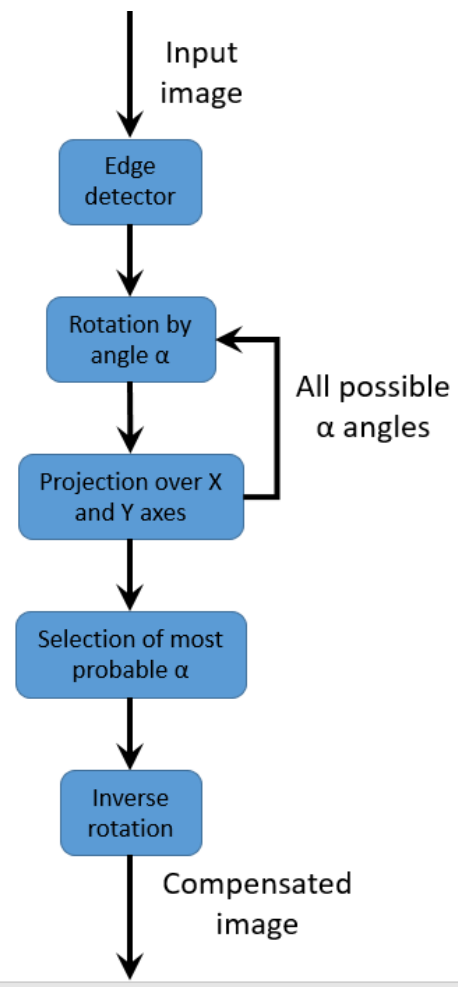

Fig. 3 Block diagram of the proposed method, parallel planes case

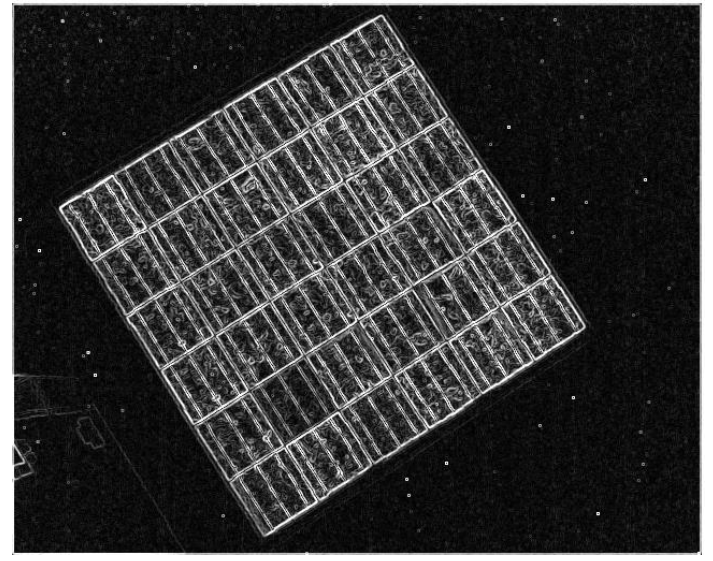

Fig. 4 Sobel edge detector of the image in Fig. 2

The various stages are detailed in the following subsections:

\section{A. Edge image computation}

The idea is here simply to reduce the amount of data to the relevant one for the next stages of the method. The first approach chosen to compute edge detection is a Sobel edge detector, as shown in Fig. 4.

\section{B. Rotation and Projection}

To cover all angles, we first rotate the edge image by values ranging from $-45^{\circ}$ until $+45^{\circ}$ (this step could be optimized with some more advanced parameter search method). For each of the obtained rotated images, the pixel values are squared and projected (summed) on the $\mathrm{X}$ and $\mathrm{Y}$ axes. An example of the resulting figures is illustrated in Fig. 5. 

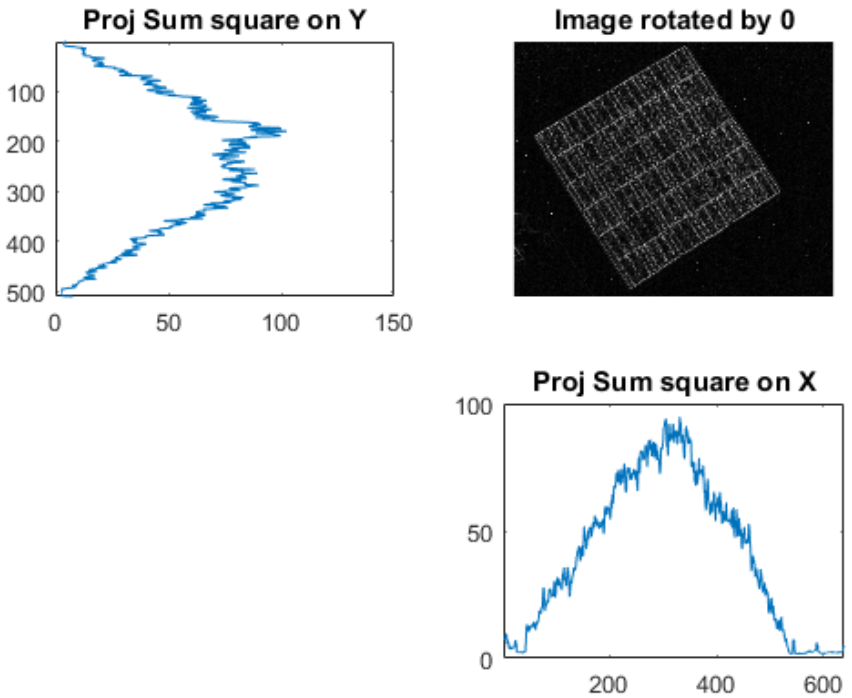

Fig. 5 Example of projections over $\mathrm{X}$ and $\mathrm{Y}$ axes for $\alpha=0^{\circ}$

\section{Selection and Compensation}

The local maxima of the projections over $\mathrm{X}$ and $\mathrm{Y}$ axes are obtained when the tested angle of the rotation corresponds to a rectified image. This is illustrated in Fig. 6.
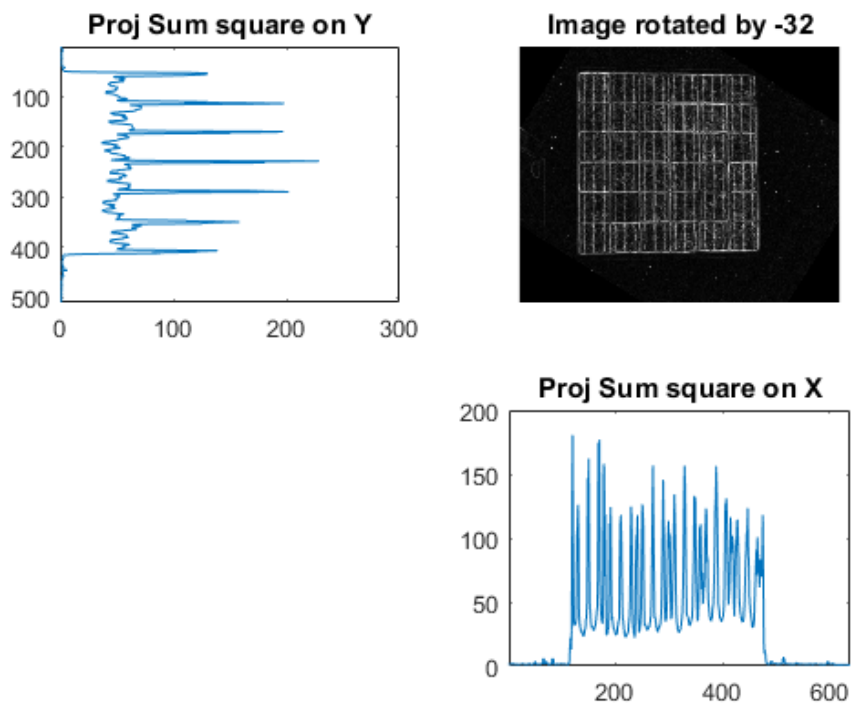

Fig. 6 Example of projections over $\mathrm{X}$ and $\mathrm{Y}$ axes for $\alpha=32^{\circ}$

The image is then rotated by the inverse of the estimated angle $\alpha$. For the chosen example, the resulting image is illustrated in Fig. 7.

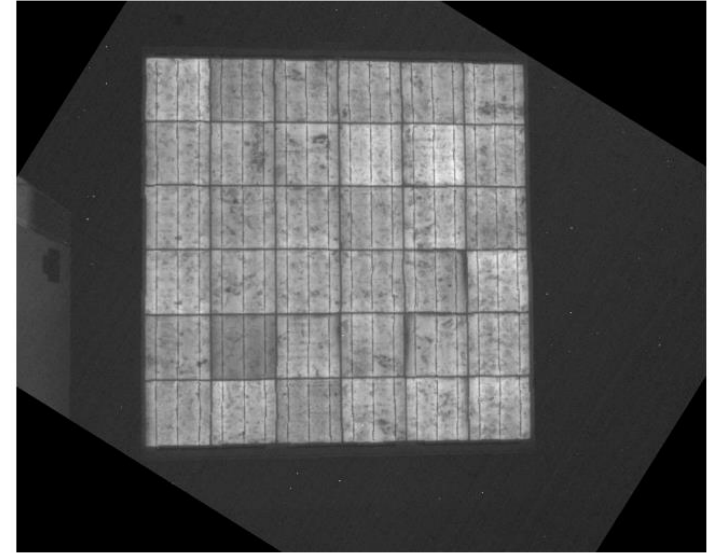

Fig. 7 Rotated version of Fig. 2 according to the estimated $\alpha$ angle

\section{Proposed Method for CASe 2- Non-Parallel Planes}

The assumption is that in that case $\mathbf{H}$ has 8 degrees of freedom. Since in homogenous coordinates, a point is defined by two degrees of freedom, knowing the projection of the four corners of the considered panel is enough to fully determine the homography $\mathbf{H}$.

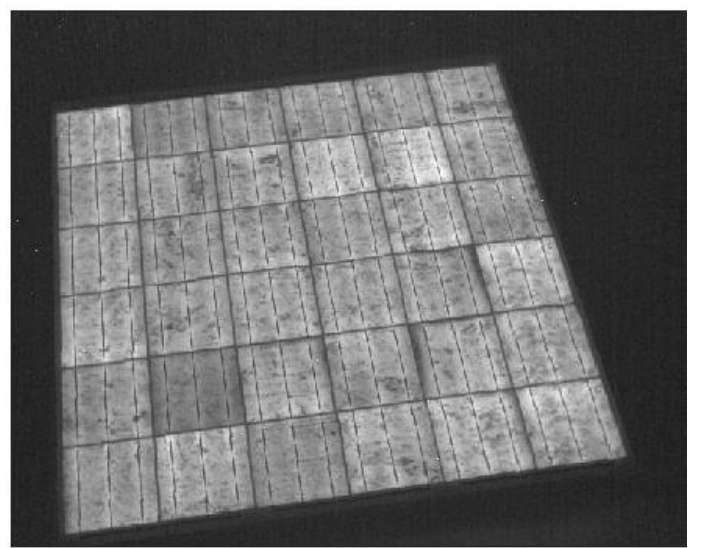

Fig. 8 Example of an input image with perspective distortion

\section{A. Border and Corner detection}

The first step for this method is therefore to detect the four corners of the panel.

A preprocessing step is applied to the image. It consists in applying the Sobel gradient followed by two morphological openings (i.e. erosion followed by dilation) to remove some of the noise: one with a horizontal line and one with vertical line, both 3 pixel long.

Then, the rotation and projection system described in Section III.B, allows identifying the four lines forming the borders of the panel. Each of the four corners can then be defined as an intersection between two of those lines. The resulting lines are illustrated in Fig. 9. 


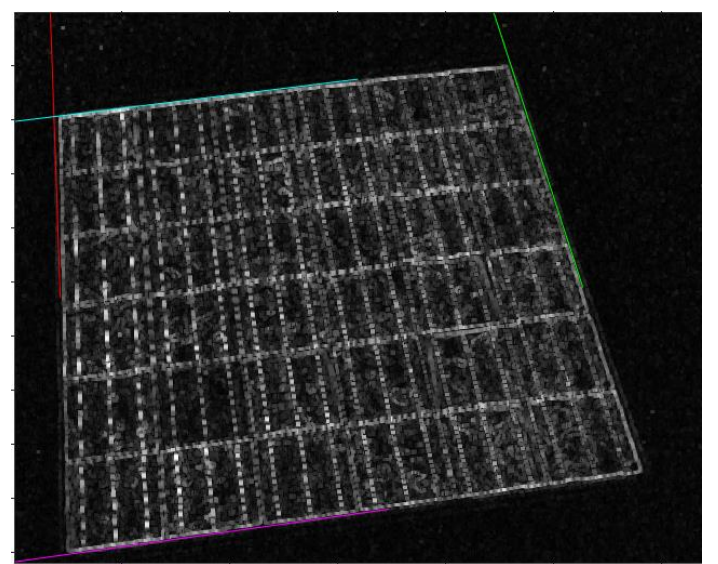

Fig. 9 Detection of the lines forming the borders of the panel

\section{B. Homography Estimation}

The Direct Linear Transform (DLT) algorithm [7] is used to estimate the homography, using the code provided by the authors from [7]. In homogenous coordinates for each couple of points $(\mathrm{Ci}, \mathrm{Di})$ :

$$
\left[\begin{array}{l}
x_{D i} \\
y_{D i} \\
z_{D i}
\end{array}\right]=\left[\begin{array}{lll}
h_{11} & h_{12} & h_{13} \\
h_{21} & h_{22} & h_{23} \\
h_{31} & h_{32} & h_{33}
\end{array}\right]\left[\begin{array}{l}
x_{C i} \\
y_{C i} \\
z_{C i}
\end{array}\right]
$$

In the image plan $\left(x_{D i}^{\prime}=x_{D i} / z_{D i}\right.$ and $\left.y^{\prime}{ }_{D i}=y_{D i} / z_{D i}\right)$

$$
\begin{aligned}
x_{D i}^{\prime} & =\frac{h_{11} x_{C i}+h_{12} y_{C i}+h_{13} z_{C i}}{h_{31} x_{C i}+h_{32} y_{C i}+h_{33} z_{C i}} \\
y_{D i}^{\prime} & =\frac{h_{21} x_{C i}+h_{22} y_{C i}+h_{23} z_{C i}}{h_{31} x_{C i}+h_{32} y_{C i}+h_{33} z_{C i}}
\end{aligned}
$$

Setting $z_{C i}=1$ (meaning that $x_{C i}$ and $y_{C i}$ are the coordinates in the image plan) and rearranging:

$x_{D i}^{\prime}\left(h_{31} x_{C i}+h_{32} y_{C i}+h_{33}\right)-\left(h_{11} x_{C i}+h_{12} y_{C i}+h_{13}\right)=0$

$y_{D i}^{\prime}\left(h_{31} x_{C i}+h_{32} y_{C i}+h_{33}\right)-\left(h_{21} x_{C i}+h_{22} y_{C i}+h_{23}\right)=0$

Which can in turn be rearranged in the form:

$$
\begin{aligned}
& a_{x c i}^{T} h=0 \\
& a_{y c i}^{T} h=0
\end{aligned}
$$

With $h=\left(h_{11}, h_{12}, h_{13}, h_{21}, h_{22}, h_{23}, h_{31}, h_{32}, h_{33}\right)^{T}$

And $\mathrm{a}_{x}=\left(-x_{C i},-y_{C i},-1,0,0,0, x_{D i}^{\prime} x_{C i}, x_{D i}^{\prime} y_{C i}, x_{D i}^{\prime}\right)^{T}$

This can in turn be summarized for all $(\mathrm{Ci}, \mathrm{Di})$ points with $\mathrm{i}$ $\epsilon\{1,2,3,4\}$ in

$$
A h=0
$$

Solving for $h$ is then linearly possible since the rank of A is 8 (we know that no three corners are collinear) and we can fix $\mathrm{h}_{33}=1$.

Practically, it is more convenient to compute the inverse of $\mathrm{H}$, i.e. the location where each pixel of the rectified image maps into the distorted image. Interpolation can then be used to estimate the value of each pixel of the rectified image. Fig.
10 illustrates the resulting mapping of Fig. 8 using bilinear interpolation.

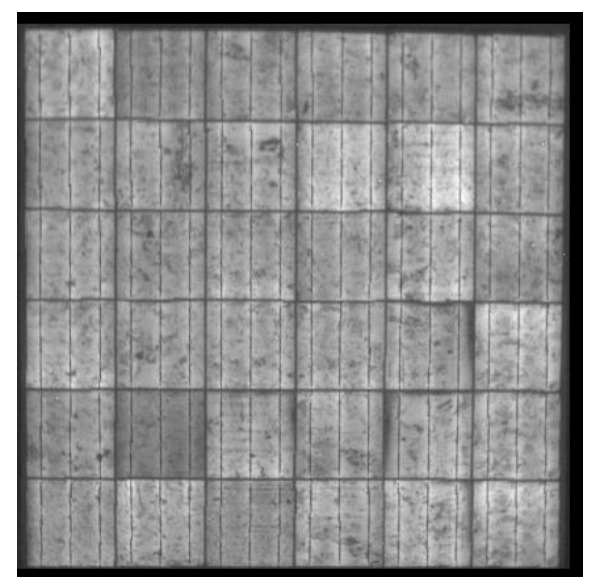

Fig. 10 Result of homography applied to Fig. 8

\section{PERFORMANCE EVALUATION}

To test the method presented here, two sets of images were acquired, corresponding to the two cases detailed above. The first one was acquired while holding the camera orthogonally to the panel and rotating it, and it consists of 54 images. The second dataset was taken while randomly positioning the camera to image the panel and consists of 254 images.

The panel imaged is a Solvis SV36-150 PV panel. The images were acquired indoor using a Photonic Science SWIR HR InGaAs which has a resolution of $640 \times 512$ and a bitdepth of 16 bits.

As those datasets were acquired using a fixed panel and a moving handheld camera, the analysis of the results has been done by visually checking that the perspective distortion was indeed corrected for.

Visual inspection shows that indeed the algorithm functions satisfyingly on all of the 308 images.

\section{DISCUSSION AND FUTURE WORK}

One identified shortcoming of the method proposed here is the assumption that the considered PV panel constitutes the main structure of the image. However, in outdoor installations, the panels are set up as arrays. Therefore, to be adapted to the array case, the proposed method needs to be preceded by a panel identification and segmentation step.

Another limitation of the method originates in the difference level of acquisition noise between indoor and outdoor imaging. The stronger noise present in outdoor images might impact the edge detection step and following projection. In this paper a "denoising" step consisting of two morphological openings on the Sobel gradient image is used, it can be replaced by another denoising method to improve the robustness.

In the same idea, averaging is often used in outdoor EL imaging of PV panels to remove the noise and improve image 
quality. In the context of a moving imaging system, for example embedded on a UAV, the required precision is likely to be smaller than one pixel, as presented here. Therefore other types of interpolation could be investigated.

One notable aspect of the method presented here is that it can be transposed to other imaging modalities for PV inspection. The transposition is immediate in the case of infrared imaging or for the luma component of visible light images.

\section{ACKNOWLEDGEMENT}

This research has been carried in the framework of the project "DronEL - Fast and accurate inspection of large photovoltaic plants using aerial drone imaging" funded by Innovation Fund Denmark under the agreement 6154-00012B.

\section{REFERENCES}

[1] R. Tsao, "Strong Chinese Market to Push Annual Global Photovoltaic Demand Above 100 Gigawatts for 2017," EnergyTrend, 2017.

[2] "Taking Flight -Civilian Drones," The Economist, 2017.

[3] C. Buerhop-Lutz et al., "IR-Imaging a Tracked PV-Plant Using an Unmanned Aerial Vehicle," in 32nd European Photovoltaic Solar Energy Conference and Exhibition, 2016, pp. 2016-2020.

[4] L. Stoicescu, M. Reuter, and J. . Werner, "Daysy: Luminescence Imaging of Pv Modules in Daylight," 29th Eur. Photovoltaics Sol. Energy Conf. Exhib. Amsterdam, Netherlands, 2014.

[5] G. Alves dos Reis Benatto et al., "Development of outdoor luminescence imaging for drone-based PV array inspection," in 44th IEEE Photovoltaic Specialists Conference, 2017, p. 6.

[6] K. Bedrich et al. "EL Imaging of PV Devices: Uncertainty due to Optical and Perspective Distortion," in 31st European Photovoltaic Solar Energy Conference and Exhibition, 2015

[7] R. Hartley and A. Zisserman, "Multiple View Geometry in Computer Vision”, Cambridge Univ. Press, 2003 